\title{
LLDAS is an attainable treat-to-target goal in childhood-onset SLE
}

\author{
Mohamed Javad Wahadat (10 , , ${ }^{1,2}$ Lotte van den Berg, ${ }^{2}$ Demi Timmermans, ${ }^{2}$ \\ Kevin van Rijswijk, ${ }^{2}$ Annette van Dijk-Hummelman, ${ }^{2}$ Susan Bakx, ${ }^{2}$ \\ Marleen Verkaaik, ${ }^{2}$ Marjan A Versnel, ${ }^{1}$ Sylvia Kamphuis ${ }^{2}$
}

To cite: Wahadat MJ, van den Berg L, Timmermans D, et al. LLDAS is an attainable treat-to-target goal in childhood-onset SLE. Lupus Science \& Medicine 2021;8:e00571. doi:10.1136/ lupus-2021-000571

- Additional supplemental material is published online only. To view, please visit the journal online (http://dx.doi.org/10. 1136/lupus-2021-000571).

MJW and LvdB are joint first authors.

Received 30 August 2021 Accepted 12 November 2021

D) Check for updates

(c) Author(s) (or their employer(s)) 2021. Re-use permitted under CC BY-NC. No commercial re-use. See rights and permissions. Published by BMJ.

'Department of Immunology, Erasmus Medical Center, Rotterdam, The Netherlands ${ }^{2}$ Department of Paediatric Rheumatology, Erasmus MC Sophia Children's Hospital, Rotterdam, The Netherlands

Correspondence to Dr Sylvia Kamphuis, SP2430 Paediatric Rheumatology, Erasmus MC Sophia Children's Hospital, Rotterdam, Netherlands; s.kamphuis@ erasmusmc.nl

\section{ABSTRACT}

Objectives To study whether clinical remission (CR) and Low Lupus Disease Activity State (LLDAS) are achievable goals in childhood-onset SLE.

Methods Data on medication use and disease activity were prospectively collected. LLDAS was defined as Safety of Estrogen in Lupus Erythematosus National AssesmentSLE disease Activity Index (SELENA-SLEDAI) $\leq 4$ with zero scores for renal, Central Nervous System (CNS), serositis, vasculitis and constitutional components, no increase in any SLEDAl component since the previous visit, PGA $\leq 1$, and prednisone dose $\leq 7.5 \mathrm{mg} /$ day. CR on treatment (Tx) was defined as a Physician Global Assessment $<0.5$, SELENA-SLEDAl $=0$, with prednisone $\leq 5 \mathrm{mg} /$ day and maintenance treatment with immunosuppressives. CR off Tx was the same but without prednisone or other immunosuppressive usage.

Results 51 patients ( 700 visits) were included. Within 3 months after diagnosis, $94.1 \%$ of children were treated with hydroxychloroquine and $60.8 \%$ with prednisone. Prednisone dosage decreased from a median of $0.74 \mathrm{mg} /$ $\mathrm{kg} /$ day at diagnosis to $0.44 \mathrm{mg} / \mathrm{kg} / \mathrm{day}$ at 3 months and $0.16 \mathrm{mg} / \mathrm{kg} /$ day at 6 months after diagnosis. Use of mycophenolate mofetil increased from $25.5 \%$ to $56.9 \%$ within 6 months after diagnosis. All children achieved LLDAS (median 186 days) and $72.5 \%$ remained in LLDAS $>50 \%$ of time. $52.9 \%$ children achieved CR on Tx, and only $21.6 \%$ children achieved CR off Tx.

Conclusions LLDAS is an attainable treat-to-target goal in contrast to CR on and off Tx. Even more, LLDAS can be reached with limited use of corticosteroids with early introduction of immunosuppressives.

\section{INTRODUCTION}

Childhood-onset SLE (cSLE) has a more aggressive disease course than adult-onset SLE, characterised by a higher frequency and severity of organ involvement and damage accrual. ${ }^{1}$ Timely achievement of disease remission is important as ongoing disease activity is associated with lower health-related quality of life (HRQOL), development of damage and increased mortality. Treatto-target has shown to be an effective strategy in various diseases and consists of strictly adjusting treatment until a specific predefined target is achieved. ${ }^{2}$ This target is most often disease remission, but can also be the lowest possible disease activity when remission is unobtainable.

\section{Key messages}

What is already known about this subject?

- Low Lupus Disease Activity State (LLDAS) in adultonset SLE is associated with less flares and damage accrual. To date, no data are published on the achievability and applicability of LLDAS in the clinical management of children with lupus.

What does this study add?

- This is the first study which shows that LLDAS can be reached in all children with SLE. LLDAS is reached in a median of 6 months with limited use of corticosteroids and the majority of patients remain in LLDAS $>50 \%$ of time.

How might this impact on clinical practice or future developments?

- Targeting LLDAS while using a strict corticosteroidsparing regimen is a feasible treat-to-target strategy in the clinical management of childhood-onset SLE and will likely decrease damage accrual and improve health-related quality of life in children with SLE.

Recently, definitions of remission for SLE and recommendations for a treat-to-target strategy were described. ${ }^{3-5}$ Complete remission without receiving treatment was a stringent goal and was only achieved in less than $3 \%$ of patients with adult-onset SLE. ${ }^{6}$ Low Lupus Disease Activity State (LLDAS) allows (low) levels of disease activity in patients who are on stable treatment and low-dose prednisone, and was an achievable target in adult-onset SLE. ${ }^{5}$ Moreover, reaching LLDAS was associated with less damage accrual, reduced flare frequency, corticosteroid sparing and improved HRQOL. ${ }^{7}$ Currently, studies investigating LLDAS as a treat-to-target strategy in cohorts of children with SLE are lacking. This study investigated whether clinical remission (CR) and LLDAS are attainable goals in a welldefined longitudinal cSLE cohort.

\section{METHODS}

Patient characteristics

Patients fulfilled the revised American College of Rheumatology classification criteria or 
the Systemic Lupus International Collaborating Clinics (SLICC) classification criteria, were diagnosed with SLE before 18 years of age and followed for at least 6 months (180 days) at the Erasmus Medical Center. All patients were diagnosed in our hospital and included at diagnosis in our prospective cohort study. Demographics, clinical characteristics and history of medication were prospectively collected from the date of diagnosis and during each follow-up visit using an electronic-Case Record File (eCRF). Disease activity was assessed by Safety of Estrogen in Lupus Erythematosus National Assesment-SLE disease Activity Index (SELENA-SLEDAI), British Isles Lupus Assessment Group (BILAG-2004) and Physician Global Assessment (PGA) at each visit. Damage was measured at the last visit using the SLICC Damage Index (SDI). ${ }^{8}$

\section{Definition of CR and LLDAS}

Based on the Definitions of Remission in SLE (DORIS) framework, for each visit 'CR off treatment' (CR off Tx) was defined as a PGA $<0.5$, SELENA-SLEDAI $=0$, without prednisone or usage of other immunosuppressives. 'CR on treatment' (CR on Tx) was defined similarly, but allowed prednisone $\leq 5 \mathrm{mg} /$ day and maintenance treatment with other immunosuppressives. ${ }^{4}$ LLDAS was defined as a SELENA-SLEDAI $\leq 4$ with zero scores for renal, Central Nervous System (CNS), serositis, vasculitis and constitutional components, without an increase in any SLEDAI component since the previous visit, with a PGA $\leq 1$ and prednisone dosage $\leq 7.5 \mathrm{mg} /$ day. Immunosuppressives were allowed for LLDAS. Hydroxychloroquine treatment was allowed for all three definitions of disease remission.

\section{Statistics}

See online supplemental methods.

\section{RESULTS}

\section{Patient characteristics}

Fifty-one patients with cSLE were included within a total of 700 visits. The median follow-up per patient was $3.0(0.5-$ $12.0)$ years with median number of visits of 12 (4-43). Median disease activity at diagnosis was 8.0 (1-29) for the SELENA-SLEDAI, $2.0(0.5-3.0)$ for the PGA and 10 (3-42) for the BILAG-2004. The most common involved organ systems at diagnosis according to the BILAG-2004 were the mucocutaneous domain $(47.1 \%)$, followed by the constitutional $(41.2 \%)$ and renal domain $(27.5 \%)$, respectively. The median number of organ systems involved at diagnosis was $3 .{ }^{1-6}$ The median SDI score at the last visit was $0(0-1)$ (online supplemental table 1).

\section{Medication usage from diagnosis to last follow-up}

In our cohort and together with hydroxychloroquine usage $(100 \%)$, prednisone $(72.5 \%)$ and mycophenolate mofetil (MMF) (62.7\%) were the most prescribed drugs. Within 3 months after diagnosis, $94.1 \%$ of the cSLE cases were on hydroxychloroquine and $60.8 \%$ used prednisone. Prednisone dosage decreased over time from a median of $0.74(0.05-1.68) \mathrm{mg} / \mathrm{kg} /$ day at diagnosis

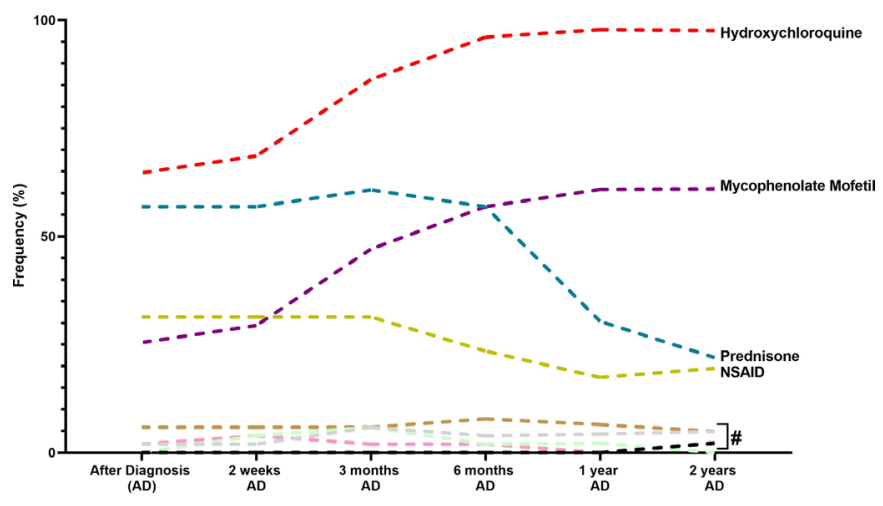

Figure 1 Medication use over time. \# indicates the lines for methotrexate, azathioprine, cyclophosphamide, rituximab and belimumab. NSAID, Non-Steroidal Anti-Inflammatory Drug.

to $0.44(0.05-1.46) \mathrm{mg} / \mathrm{kg} /$ day at 3 months and 0.16 $(0.05-1.54) \mathrm{mg} / \mathrm{kg} / \mathrm{day}$ at 6 months after diagnosis. This decrease was paralleled by an increased usage of MMF (25.5\% at diagnosis, $47.1 \% 3$ months and $56.9 \% 6$ months after diagnosis) (figure 1). Additional information is provided in online supplemental table 2.

\section{Disease remission}

LLDAS was achieved in all patients within a median time of 186 (28-846) days. Patients remained in this state for 693 (78-2676) days. Moreover, we assessed the cumulative time spent in LLDAS, since many studies have reported a 50\% reduction in damage accrual in patients who have remained in LLDAS for $50 \%$ of their follow-up time. ${ }^{910}$ The majority $(72.5 \%)$ of patients were in LLDAS for $>50 \%$ of the follow-up time. At follow-up visits of patients not in LLDAS, the haematological, mucocutaneous and renal domains were most often still active according to the BILAG and SELENA-SLEDAI. CR on Tx was ever achieved in $52.9 \%$ of the patients within a median time of 415 (40-1585) days. These patients spent only 176 (35-1198) days of the total follow-up time in this state. Only four patients were able to stay in this state for $>50 \%$ of the follow-up time. CR off Tx was achieved in $21.6 \%$ of patients within a median time of 536 (51-2103) days. These patients spent 176 (98-945) days of the total follow-up time in this state. Only one patient remained in this state for $>50 \%$ of the follow-up time (table 1 ).

\section{Factors related to reaching LLDAS}

Patients who achieved LLDAS within 6 months had lower SELENA-SLEDAI and lower PGA scores at diagnosis than those patients who did not achieve LLDAS (online supplemental figure 1). Univariate analysis showed that SELENA-SLEDAI at diagnosis, PGA at diagnosis and prednisone dose at 3 months were associated with reaching LLDAS within 6 months. In the multivariate model, prednisone dose and MMF usage at 3 months were both associated with reaching LLDAS within 6 months. Here, a higher dose of prednisone at 3 months was associated with not reaching LLDAS within 6 months, while the use 
Table 1 LLDAS and clinical remission

\begin{tabular}{|c|c|c|c|}
\hline $\mathrm{n}=51 ; 700$ visits, median follow-up 1106 days & LLDAS & CR on Tx & CR off Tx \\
\hline Status ever achieved & $51(100 \%)$ & $27(52.9 \%)$ & $11(21.6 \%)$ \\
\hline Time to status in days, median (range) & $186(28-846)$ & $415(40-1585)$ & $536(51-2103)$ \\
\hline Time in status in days, median (range) & $693(78-2676)$ & $176(35-1198)$ & $176(98-945)$ \\
\hline$\%$ of time in status during follow-up, median (range) & $72.1(11.6-98.5)$ & $17.9(5-73.6)$ & $17.4(4.1-88.6)$ \\
\hline Number $(\%)$ of patient in status $>50 \%$ of follow-up time & $37(72.5)$ & $4(14.8)$ & $1(9.1)$ \\
\hline $\begin{array}{l}\text { Organ domains still active at visits not in LLDAS } \\
\text { ( } N=195 / 700 \text { visits) }\end{array}$ & BILAG-2004 & & SELENA-SLEDAI \\
\hline Constitutional & $36(18.5 \%)$ & Constitutional & $19(9.7 \%)$ \\
\hline Mucocutaneous & $110(56.4 \%)$ & Mucocutaneous & $119(61.0 \%)$ \\
\hline Neuropsychiatric & $2(1.0 \%)$ & Neuropsychiatric & $1(0.5 \%)$ \\
\hline Musculoskeletal & $55(28.2 \%)$ & Musculoskeletal & $15(7.7 \%)$ \\
\hline Cardiorespiratory & $19(9.7 \%)$ & Cardiorespiratory & $11(5.6 \%)$ \\
\hline Renal & $91(46.7 \%)$ & Renal & $112(57.4 \%)$ \\
\hline Haematological & $123(63.1 \%)$ & Haematological & $160(82.1 \%)$ \\
\hline Gastrointestinal & $15(7.7 \%)$ & Immunological & $81(41.5 \%)$ \\
\hline Ophthalmological & $3(1.5 \%)$ & Ophthalmological & $0(0 \%)$ \\
\hline
\end{tabular}

SELENA-SLEDAI domains are scored accordingly: constitutional (fever), mucocutaneous (vasculitis, rash, alopecia, mucosal ulcers), neuropsychiatric (psychosis, seizure, organic brain syndrome, cranial nerve disorders, lupus headache, CVA), musculoskeletal (arthritis, myositis), cardiorespiratory (pleurisy, pericarditis), renal (urinary casts, haematuria, proteinuria, pyuria), haematological (low complement, thrombocytopenia, leucopenia), immunological (increased DNA binding), ophthalmological (visual disturbance).

BILAG, British Isles Lupus Assessment Group; CR, clinical remission; LLDAS, Low Lupus Disease Activity State; SELENA-SLEDAI, Safety of Estrogen in Lupus Erythematosus National Assesment-SLE disease Activity Index; Tx, treatment.

of MMF at 3 months was associated with reaching LLDAS within 6 months (table 2).

\section{DISCUSSION}

Previous work has shown that the majority of adults with cSLE do not achieve medication-free remission, develop significant damage at young age and have impaired HRQOL. ${ }^{11}$ Being in LLDAS and CR was associated with significant reduction of damage in adult-onset SLE. ${ }^{9}$ Failure to achieve LLDAS within 6 months after diagnosis was associated with early damage accrual. ${ }^{12}$ In this study, we show that LLDAS is an achievable goal in cSLE with a

Table 2 Binary logistic regression analysis of variables associated with achieving LLDAS at 6 months as outcome measure

\begin{tabular}{|c|c|c|c|c|c|c|}
\hline & \multicolumn{3}{|c|}{ Univariate } & \multicolumn{3}{|c|}{ Multivariate } \\
\hline & B & $P$ value & Exp & B & $P$ value & Exp \\
\hline Age at diagnosis & -0.014 & 0.887 & 0.986 & & & \\
\hline Gender & -0.047 & 0.952 & 0.955 & & & \\
\hline SLEDAI at Dx & -0.14 & 0.024 & 0.869 & -0.076 & 0.485 & 0.927 \\
\hline PGA at Dx & -0.787 & 0.048 & 0.455 & -0.179 & 0.86 & 0.836 \\
\hline Number of domains involved & -0.323 & 0.103 & 0.724 & & & \\
\hline SDI score at last visit & -1.099 & 0.217 & 0.333 & & & \\
\hline $\mathrm{HCQ}$ use at 3 months & -0.777 & 0.537 & 0.46 & 0.461 & 0.746 & 1.585 \\
\hline Prednisone dose at 3 months & -2.729 & 0.042 & 0.065 & -3.421 & 0.041 & 0.033 \\
\hline
\end{tabular}

A cut-off of $p<0.100$ was set to select the variables for the multivariate logistic regression.

BILAG, British Isles Lupus Assessment Group; Dx, diagnosis; HCQ, hydroxychloroquine; LLDAS, Low Lupus Disease Activity State; MMF, mycophenolate mofetil; PGA, Physician Global Assessment; SDI, SLICC Damage Index; SLEDAI, SLE disease Activity Index; SLICC, Systemic Lupus International Collaborating Clinics. 
chosen treatment strategy, which included limited use of prednisone. Furthermore, patients with cSLE remained in LLDAS for the majority of their follow-up time. The follow-up in our study is too short to make reliable conclusions regarding the relation between LLDAS and damage accrual.

Evidence-based guidelines on initiation, tapering and cessation of corticosteroids do not exist for the management of SLE. ${ }^{13}$ Important differences in corticosteroid usage for cSLE treatment exist between medical centres, which are not associated with disease activity. ${ }^{14}$ Clinicians are reluctant to taper corticosteroids as this increases the chance of a lupus flare. ${ }^{15}$ In our centre, we have a strict corticosteroid-sparing treatment strategy to limit the well-known detrimental side effects of corticosteroids on damage accrual, including growth failure and HRQOL in children with SLE. In general, prednisone without other immunosuppressives is allowed for 3 months, after which other immunosuppressives are added to allow corticosteroid tapering. Here we show that within 6 months after diagnosis, prednisone dosages can safely be reduced in the majority of patients with cSLE when paralleled by increased use of immunosuppressives. Regression analysis revealed that a higher prednisone dose at 3 months was associated with not reaching LLDAS within 6 months, while MMF use at 3 months was associated with the opposite. This highlights the effectivity of this strategy, which is consistent with the international consensus that indicates that immunosuppressives should be considered when prednisone dosages remain above $0.15 \mathrm{mg} / \mathrm{kg} /$ day. ${ }^{16}$

Patients less prone to reach LLDAS within 6 months in general had a higher SELENA-SLEDAI and PGA scores at diagnosis, but the BILAG score was not discriminative. This is most likely explained by the fundamental differences between the scoring systems. The BILAG scores all organ domains equally, which is in contrast to the SELENA-SLEDAI. For example, renal activity, an organ system known for needing considerable time to become inactive, has a relative large contribution to the total SELENA-SLEDAI score. ${ }^{17}$

This study has limitations. The size of our cohort is relatively small and therefore our results need to be validated in additional studies using larger cohorts of patients with cSLE. However, the detailed clinical data available from every patient since diagnosis, collected prospectively at each follow-up visit and the uniform treatment approach are strengths of this study and support the robustness of the conclusions drawn.

In conclusion, LLDAS is an achievable treat-to-target goal in cSLE and can be reached with limited use of corticosteroids with early introduction of immunosuppressives.

Acknowledgements The authors thank all the patients with CSLE who participated.

Contributors Conceptualisation-MJW, MAV and SK. Acquisition of data-MJW, LvdB, DT, KvR, AvD, SB, MV and SK. Data analysis-MJW, LvdB, MAV and SK. Interpretation-MJW, LvdB, MAV and SK. Drafting of the manuscript-MJW, LvdB, MAV and SK. All authors revised the manuscript critically and approved the final version.
Funding This work was made possible by the support of the Sophia Children's Hospital Fund, NVLE (Dutch patient organisation for Lupus, APS, Scleroderma and MCTD) and Dutch Arthritis Society. The research was performed within the framework of the Erasmus Postgraduate School Molecular Medicine.

Competing interests None declared.

Patient consent for publication Parental/guardian consent obtained.

Ethics approval The Erasmus Medical Center Ethics Committee (MEC-2019-0412) approved the study and written informed consent was obtained from patients and/ or parents.

Provenance and peer review Not commissioned; externally peer reviewed.

Author note please add my orcid ID as well: sylvia kamphuis orcid ID 0000-00021964-352X

Supplemental material This content has been supplied by the author(s). It has not been vetted by BMJ Publishing Group Limited (BMJ) and may not have been peer-reviewed. Any opinions or recommendations discussed are solely those of the author(s) and are not endorsed by BMJ. BMJ disclaims all liability and responsibility arising from any reliance placed on the content. Where the content includes any translated material, BMJ does not warrant the accuracy and reliability of the translations (including but not limited to local regulations, clinical guidelines, terminology, drug names and drug dosages), and is not responsible for any error and/or omissions arising from translation and adaptation or otherwise.

Open access This is an open access article distributed in accordance with the Creative Commons Attribution Non Commercial (CC BY-NC 4.0) license, which permits others to distribute, remix, adapt, build upon this work non-commercially, and license their derivative works on different terms, provided the original work is properly cited, appropriate credit is given, any changes made indicated, and the use is non-commercial. See: http://creativecommons.org/licenses/by-nc/4.0/.

ORCID iD

Mohamed Javad Wahadat http://orcid.org/0000-0003-3961-1274

\section{REFERENCES}

1 Kamphuis S, Silverman ED. Prevalence and burden of pediatriconset systemic lupus erythematosus. Nat Rev Rheumatol 2010;6:538-46.

2 Atar D, Birkeland KI, Uhlig T. 'Treat to target': moving targets from hypertension, hyperlipidaemia and diabetes to rheumatoid arthritis. Ann Rheum Dis 2010;69:629-30.

3 van Vollenhoven RF, Mosca M, Bertsias G, et al. Treat-to-target in systemic lupus erythematosus: recommendations from an international Task force. Ann Rheum Dis 2014;73:958-67.

4 van Vollenhoven R, Voskuyl A, Bertsias G, et al. A framework for remission in SLE: consensus findings from a large international Task force on definitions of remission in SLE (DORIS). Ann Rheum Dis 2017;76:554-61.

5 Franklyn K, Lau CS, Navarra SV, et al. Definition and initial validation of a lupus low disease activity state (LLDAS). Ann Rheum Dis 2016;75:1615-21.

6 Steiman AJ, Urowitz MB, Ibañez D, et al. Prolonged clinical remission in patients with systemic lupus erythematosus. J Rheumatol 2014;41:1808-16.

7 Smith EMD, Gorst SL, Al-Abadi E, et al. "It is good to have a target in mind": Qualitative views of patients and parents informing a treat to target clinical trial in JSLE. Rheumatology 2021:keab173.

8 Gladman D, Ginzler E, Goldsmith C, et al. The development and initial validation of the systemic lupus international collaborating Clinics/American College of rheumatology damage index for systemic lupus erythematosus. Arthritis Rheum 1996;39:363-9.

9 Petri M, Magder LS. Comparison of remission and lupus low disease activity state in damage prevention in a United States systemic lupus erythematosus cohort. Arthritis Rheumatol 2018;70:1790-5.

10 Golder V, Kandane-Rathnayake R, Huq M, et al. Lupus low disease activity state as a treatment endpoint for systemic lupus erythematosus: a prospective validation study. Lancet Rheumatol 2019;1:e95-102.

11 Groot N, Shaikhani D, Teng YKO, et al. Long-Term clinical outcomes in a cohort of adults with childhood-onset systemic lupus erythematosus. Arthritis Rheumatol 2019;71:290-301.

12 Piga M, Floris A, Cappellazzo G, et al. Failure to achieve lupus low disease activity state (LLDAS) six months after diagnosis is associated with early damage accrual in Caucasian patients with systemic lupus erythematosus. Arthritis Res Ther 2017;19:247. 
13 Apostolopoulos D, Morand EF. It hasn't gone away: the problem of glucocorticoid use in lupus remains. Rheumatology 2017;56:i114-22.

14 Brunner HI, Klein-Gitelman MS, Ying J, et al. Corticosteroid use in childhood-onset systemic lupus erythematosus-practice patterns at four pediatric rheumatology centers. Clin Exp Rheumatol 2009;27:155-62.

15 Tani C, Elefante E, Signorini V, et al. Glucocorticoid withdrawal in systemic lupus erythematosus: are remission and low disease activity reliable starting points for stopping treatment? A real-life experience. RMD Open 2019;5:e000916.

16 Hollander MC, Sage JM, Greenler AJ, et al. International consensus for provisions of quality-driven care in childhood-onset systemic lupus erythematosus. Arthritis Care Res 2013;65:1416-23.

17 Askanase AD, Merrill JT. Measuring disease activity in SLE is an ongoing struggle. Nat Rev Rheumatol 2019;15:194-5. 